\title{
Uso de dispositivos tecnológicos de los estudiantes de Fima de la Espoch, carrera de Estadística
}

\author{
Use of technological devices of the Fima students of the Espoch, Statistics \\ career
}

Johanna Enith Aguilar Reyes. ${ }^{1}$, Paulina Elizabeth Valverde Aguirre. ${ }^{2}$, Juan Efraín Sánchez Vimos. ${ }^{3} \&$ Teresa Angélica Altamirano Novillo. ${ }^{4}$

Recibido: 25-05-2019 / Revisado: 18-06-2019 /Aceptado: 30-07-2019/ Publicado: 06-09-2019

\begin{abstract}
.
DOI: https://doi.org/10.33262/cienciadigital.v3i3.3.773

The main objective of carrying out this research project is to know the use of technological devices that have the polytechnic students of the School of Physics and Mathematics career of Statistics. To do this, we took a sample of population with similar characteristics which helped us to study and with that to be able to generalize the results obtained in the study in statistical surveys, for the collection and tabulation of data, of such so that we can determine which is the device that predominates more in the young people and the consequences that consequences that of the excessive use of technological devices causing that the students desert the race. Therefore, it is necessary that society in general, but in a more direct way the family and the school, become aware of this phenomenon, assuming the responsibilities incumbent on them and providing the necessary training and mechanisms to the youngest to that these make proper and proper use of technologies.
\end{abstract}

Keywords: Students, technology, technological devices, internet, diseases.

1 Escuela Superior Politécnica de Chimborazo, Facultad de Ciencias. Riobamba, Ecuador. johannae.aguilar@espoch.edu.ec

2 Escuela Superior Politécnica de Chimborazo, Facultad de Ciencia. Riobamba, Ecuador. paulina.valverde@espoch.edu.ec

${ }^{3}$ Escuela Superior Politécnica de Chimborazo, Facultad de Ciencias. Riobamba, Ecuador. jusanchez@yahoo.es

4 Escuela Superior Politécnica de Chimborazo, Facultad de Ciencias. Riobamba, Ecuador. taltamirano@yahoo.es 


\section{Resumen.}

El principal objetivo de llevar a cabo este proyecto de investigación es conocer el uso de dispositivos tecnológicos que tienen los estudiantes politécnicos de la Escuela de Física y Matemática carrera de Estadística. Para ello tomamos una muestra de población con características similares la cual nos ayudó para el estudio y con ello poder generalizar los resultados obtenidos en la se trata en la realización de encuestas a los estudiantes de estadística, para la recolección y tabulación de datos, de tal manera que podamos determinar cuál es el dispositivo que predomina más en los jóvenes y las consecuencias que consecuencias que del uso excesivo de dispositivos tecnológicos haciendo que los estudiantes deserten la carrera. Por ello, es preciso que la sociedad en general, pero de una manera más directa la familia y la escuela, tomen conciencia de este fenómeno, asumiendo las responsabilidades que les incumben y dotando de la formación y de los mecanismos necesarios a los más jóvenes para que estos hagan un uso correcto y adecuado de las tecnologías

Palabras claves: Estudiantes, tecnología, dispositivos tecnológicos, internet, enfermedades.

\section{Introducción.}

Actualmente vivimos en una sociedad que se caracteriza, entre otros aspectos, por su carácter de globalidad y por el ritmo vertiginoso de sus cambios.

La rapidez con que estamos sometidos a los avances tecnológicos hace que cualquier logro en este campo se quede obsoleto en un plazo de dos o tres años y sea ampliamente superado por nuevos instrumentos. En una sociedad tan cambiante y en la que los valores son tan a menudo minusvalorados o cuestionados es preciso actuar con prudencia $y$, a la vez, con decisión.

Por ello, es preciso que la sociedad en general, pero de una manera más directa la familia y la escuela, tomen conciencia de este fenómeno, asumiendo las responsabilidades que les incumben y dotando de la formación y de los mecanismos necesarios a los más jóvenes para que estos hagan un uso correcto y adecuado de las tecnologías para beneficiarse de los muchos aspectos positivos que conllevan y para evitar y minimizar sus riesgos.

Podemos concluir, asimismo, que nuestra sociedad avanza hacia la plena cobertura de infraestructura tecnológica, tanto en los hogares como en las escuelas.

Los adolescentes están viviendo en un entorno digitalizado que ha condicionado su manera de actuar, relacionarse y comunicarse. Este colectivo es experto en el manejo instrumental de estos soportes, pero no los suelen utilizar de un modo reflexivo. 


\section{Desarrollo.}

\section{¿Qué son los dispositivos tecnológicos?}

Los dispositivos tecnológicos los aparatos que se han ido renovando a lo largo del tiempo debido al avance de la tecnología. Estos aparatos se caracterizan por ser instrumentos que aumentan la calidad de vida de los individuos tales como los automóviles, los celulares, las computadoras y todos aquellos que sirven para satisfacer las necesidades del hombre como el entretenimiento, seguridad y estabilidad, entre otras cosas.

\section{Los jóvenes y las nuevas tecnologías}

Estudios realizados por la Fundación Pfizer, el 98\% de los jóvenes de 11 a 20 años disponen un dispositivo tecnológico. De ese porcentaje, siete de cada 10 afirman acceder a la Red por un tiempo diario de, al menos, 1.5 horas. Es, por tanto, una realidad obvia el alto grado de uso de las nuevas tecnologías entre los adolescentes y jóvenes (Abece, 2014).

Las nuevas Tecnologías de la Información y de la Comunicación (TIC) hoy en la actualidad el hombre ha disminuido considerablemente las actividades económicas. El internet es una gran facilidad para los jóvenes ya que da la mayor comodidad y facilidad para ser empleado en cada uno de los dispositivos que posee el hombre. (Small, G. y Vorgan, G. 2009)

El uso es positivo, siempre que no se dejen de lado el resto de las actividades propias de una vida normal (estudiar, hacer deporte, ir al cine, salir con los amigos o relacionarse con la familia). Otra cosa es cuando el abuso de la tecnología provoca aislamiento, induce ansiedad, afecta a la autoestima y le hace perder al sujeto su capacidad de control (Echeburúa y Corral, 2010).

El uso excesivo de los dispositivos tecnológicos puede facilitar el aislamiento del ser humano, el bajo rendimiento académico o laboral, el desinterés por otros temas y por el ocio activo, mala conducta y problemas económicos, así como el sedentarismo y la obesidad. Los problemas se extienden a todos los ámbitos de la persona afectada (salud, familia, escuela y relaciones sociales) (Echeburúa y Requesens, 2012).

¿Cómo utilizan los adolescentes los dispositivos tecnológicos?

Redes sociales, móviles de última generación, dispositivos como iPod, etc. son herramientas imprescindibles de la cultura juvenil. Es de suma importancia estudiar cómo son utilizados los dispositivos tecnológicos en los jóvenes ya que están entusiasmados con las posibilidades que les ofrece ese mundo de la tecnología, pero no está exento de riesgos. Según datos del informe de la Fundación Antena 3 (Canalda, 2010): 
Los adolescentes aumentan el tiempo de uso ante las pantallas de los dispositivos, pasando de 199 minutos en los niños de la primaria, a 261 minutos en los jóvenes de la secundaria.

Los niños y adolescentes distribuyen ese tiempo de la siguiente manera: una hora y cuarenta y nueve minutos viendo la televisión, cuarenta y ocho minutos jugando con la consola y una hora y cuarenta y cuatro minutos conectado a internet.

\section{Principios que presenta una persona con el uso excesivo de dispositivos tecnológicos}

Las principales señales de alarma que denotan una dependencia a las TIC que pueden ser un reflejo de la conversión de una afición en una adicción son las siguientes (Echeburúa y Corral, 2010):

a) No conciliar sueño por el uso constante del dispositivo tecnológico.

b) Descuidar las actividades que usualmente realizar, como el contacto con la familia, las relaciones sociales, el estudio o el cuidado de la salud.

c) Pensar en el "celular" constantemente, incluso cuando no se está conectado a una red de internet y sentirse irritado excesivamente cuando la conexión falla.

d) Aislarse socialmente.

e) Bajar el rendimiento en los estudios.

\section{Factores de riesgo ante el abuso de uso de dispositivos tecnológicos}

El uso excesivo de los dispositivos tecnológicos hoy en día es la preocupación en la sociedad por la importancia que cobran estos dispositivos en la vida diaria. El uso de los dispositivos tecnológicos puede ocasionar daños psicológicos y neurológicos. Así lo demuestra un estudio realizado por la operadora británica Sky Broadband. Los jóvenes son los que están más expuestos a los peligros, ya que el cerebro se adapta a los cambios constantes. La investigación también refleja la adicción que se puede llegar a generar por la necesidad de consultar lo que sucede en las redes sociales. Según datos de la Organización Mundial de la Salud (OMS), Salud180 (2017), una de cada cuatro personas experimenta cambios en la conducta asociados a las adicciones sin sustancias.

Sandra Espinoza, neuróloga, explica que el insomnio que produce el utilizar los dispositivos por la noche es el causante de la falta de concentración. Las horas destinadas para el sueño, según Espinoza, son el momento en que se fijan las memorias, si la persona no logra descansar adecuadamente durante varios días, su rendimiento disminuirá. Además, al no realizar actividades físicas por pasar más tiempo frente a las pantallas de los dispositivos, no se logra desarrollar la musculatura ni la motricidad fina. Por esto surgen los problemas en el aprendizaje y la falta de interés, sobre todo de las nuevas generaciones hacia otras actividades, como la lectura, y no se desarrolla su función cognitiva. 
El estrés y la ansiedad que se experimentan en los partidos de videojuegos se reflejan en enfermedades como la dermatitis e hidradenitis palmar. Así lo explica la dermatóloga Luz María Dressendorfer, quien afirma que, aunque antes $\neg$ existía esta enfermedad, en la actualidad es más común que los síntomas aparezcan a una edad más temprana. La tablet y el teclado de la computadora también pueden ser causantes de estas molestias, porque la piel adquiere sensibilidad al material al estar en contacto constante. La visión también se afecta por los videojuegos, debido a la disminución en el parpadeo, lo que ocasiona la sequedad ocular. Estudios señalan que durante una conversación se parpadea 22 veces por minuto, a diferencia de las siete veces que se parpadea mientras se está frente a una pantalla. Para Espinoza, la tecnología facilita el desarrollo de las actividades diarias, pero hay que establecer límites en su uso para prevenir daños irreparables en la salud física y mental (Sandra Espinoza, 2015).

\section{Metodología.}

En el presente capitulo se muestran los tipos, técnicas y procedimientos que fueron utilizados para llevar a cabo la investigación. Conforme a los diferentes usos que los estudiantes les dan a los dispositivos tecnológicos se han planteado recopilar información de una muestra de estudiantes a fin de conocer sus intereses, y opiniones sobre el uso de dispositivos tecnológicos y así identificar los posibles problemas que puedan presentar cada uno de ellos.

El tipo de investigación realizada corresponde a la investigación descriptiva que tiene como objetivo describir y delimitar los diferentes elementos del problema. Y a través de esta investigación podemos observar y describir la situación actual del uso que le dan los estudiantes a los dispositivos tecnológicos.

A continuación, se resume los pasos que se realizaron en el proyecto:

1. Definición y planteamiento del problema.

2. Formulación de los objetivos.

3. Selección del método de investigación.

4. Recolección de información previa para la elaboración de un posible cuestionario.

5. Planteamiento de la muestra y revisión del cuestionario previo.

6. Modificaciones al cuestionario final.

7. Aplicación del cuestionario final aprobado.

8. Tratamiento y análisis de los datos.

9. Obtención de resultados y gráficos estadísticos.

10. Revisión de los resultados y obtención de conclusiones finales. 


\section{Métodos de Investigación}

\section{Métodos Teóricos}

En esta investigación hemos utilizados los métodos teóricos que permiten describir conceptualmente al objeto y se utilizan para procesar la información teórica y la obtenida por la aplicación de los métodos empíricos.

\section{Método Descriptivo}

Tiene como propósito la observación empleada para describir la problemática tal como se presenta en la realidad del lugar a ser objeto de estudio. Este método permitirá explicar de forma detallada los posibles problemas que hayan tenido al usar dispositivos tecnológicos, a través de las encuestas que utilizaremos para llevar a cabo la recopilación de información podremos determinar los factores influyentes de los problemas y determinar una posible solución.

\section{Obtención de la Muestra}

La población de estudio son los estudiantes de la Escuela de Física y Matemáticas de la carrera de Estadística de la ESPOCH son de 119 estudiantes la cual mediante formula empleadas obtuvimos una muestra de 92 estudiantes.

\section{Técnicas de recolección de datos}

Una vez determinada la muestra se procede a la elaboración de una encuesta. Para esto se formulan preguntas referentes al problema que tengan sobre el uso de dispositivos tecnológicos. Una vez realizada la encuesta pasa a revisión por la autoridad experto en la materia, de esta manera se realizan las modificaciones que se requieran.

Una vez aprobada la encuesta por la autoridad encargada se procede a aplicar a los estudiantes de la Escuela de Física y Matemática de la carrera de la ESPOCH.

Posteriormente se realizará la base de datos que contendrá las respuestas de cada una de las encuestas y se realizaran las tablas dinámicas y gráficos que representen cada una de las preguntas planteadas en la encuesta.

Instrumentos Utilizados: Se utilizaron los siguientes instrumentos:

- Encuestas impresas

- Computador

- Calculadora 
- $\quad$ Apuntes de clases

\section{Resultados.}

Resultados obtenidos después de realizar encuestas a los estudiantes de la Escuela de Física y Matemática carrera Estadística de la ESPOCH con el propósito de evidenciar si el tema de la investigación es en realidad un problema que necesita solución y se exponga la más conveniente, para llevar a cabo esta encuesta se formuló preguntas referidas a las horas, lugares y problemas causados por el uso de dispositivos tecnológicos y en las cuales formulamos diez preguntas para poder recolectar información necesario a fin de cumplir y mostrar los resultados obtenidos.

\section{Pregunta 1}

Tabla 1. ¿Cuál es el dispositivo tecnológico que más usa usted?

\begin{tabular}{lccccc}
\hline $\begin{array}{l}\text { ¿Cuál es el dispositivo } \\
\text { tecnológico que más usa usted? }\end{array}$ & Computador & Celular & Tablet & Otros & ¿Cuál? \\
\hline $\begin{array}{l}\text { Frecuencia } \\
\%\end{array}$ & 29 & 74 & 1 & & \\
\hline & $31,52 \%$ & $80,43 \%$ & $1,09 \%$ & 0 & 0 \\
\hline
\end{tabular}

Figura 1. ¿Cuál es el dispositivo tecnológico que más usa usted?

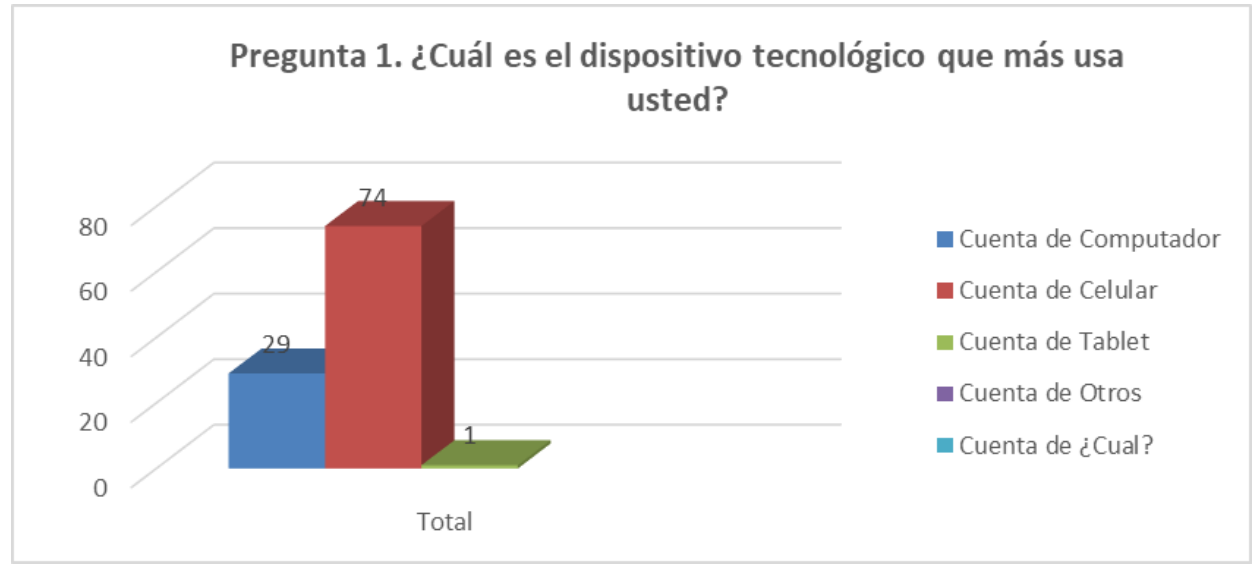

Análisis pregunta 1 ¿Cuál es el dispositivo tecnológico que más usa usted?

Según la información recopilada mediante la encuesta realizada a los estudiantes de la Escuela de Física y Matemática de la carrera de Estadística, luego de la respectiva tabulación de datos se obtuvo los siguientes resultados un $80.43 \%$ de estudiantes hacen un uso del celular, seguido del computador con un $31.52 \%$ con un $1.9 \%$ utilizan la Tablet, es decir, que el dispositivo que hacen más uso en la población estudiada es el celular con un $80.43 \%$. 
Tabla 2. ¿Considera usted que usa en exceso los dispositivos tecnológicos?

\begin{tabular}{ccc}
\hline ¿Considera usted que usa en exceso los & & \\
dispositivos tecnológicos? & frecuencia & $\%$ \\
\hline No & 33 & $35,87 \%$ \\
Si & 59 & $64,13 \%$ \\
Total general & 92 & $100,00 \%$ \\
\hline
\end{tabular}

Figura 2. ¿Considera usted que usa en exceso los dispositivos tecnológicos?

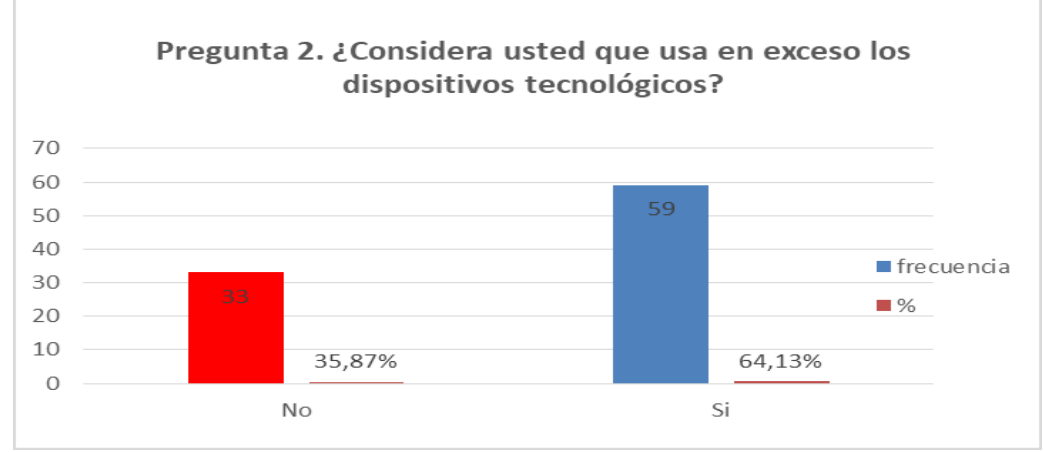

Análisis pregunta 2 ¿Considera usted que usa en exceso los dispositivos tecnológicos?

Luego de realizada la tabulación de los datos podemos notar que un $35.87 \%$ de los estudiantes no consideran que usan en exceso el dispositivo tecnológico pero un 64,13\% consideran que usan en exceso los dispositivos tecnológicos, es decir, que más del 50\% utilizan en exceso el dispositivo tecnológico.

Tabla 3. ¿Cuántas horas al día usa usted un dispositivo tecnológico?

\begin{tabular}{ccc}
\hline ¿Cuántas horas al día usa usted un dispositivo & & \\
tecnológico? & frecuencia & $\%$ \\
\hline 1 - 2 horas & 4 & $4,35 \%$ \\
2 - 4 horas & 23 & $25,00 \%$ \\
4 - 6 horas & 34 & $36,96 \%$ \\
Más de 6 horas & 31 & $33,70 \%$ \\
Total general & 92 & $100,00 \%$ \\
\hline
\end{tabular}

Figura 3. ¿Cuántas horas al día usa usted un dispositivo tecnológico?

Pregunta 3. ¿Cuantas horas al día usa usted un dispositivo tecnológico?

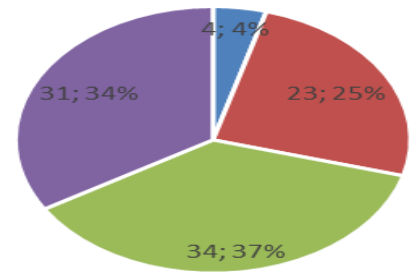

- 1 - 2 horas

- 2 - 4 horas

= 4- 6 horas

- Mas de 6 horas 
Análisis pregunta 3 ¿Cuantas horas al día usa usted un dispositivo tecnológico?

Luego de la respectiva tabulación de la pregunta tres se pudo concretar que los estudiantes con poca frecuencia que utilizan un dispositivo tecnológico son de 1-2 horas lo cual está representado con el 4,35\%, mientras lo que utilizan de 2-4 horas esta con un $25 \%$ de los estudiantes y vemos que hay una igualdad en las frecuencias de 4-6 horas y más de 6 horas con un $33,70 \%$ es decir, que los estudiantes que utilizan desde 4 horas en adelante un dispositivo tecnológico es el 70,66\%.

Tabla 4. ¿Principalmente para que usa los dispositivos tecnológicos?

\begin{tabular}{ccccc}
\hline $\begin{array}{c}\text { ¿Principalmente para que usa los } \\
\text { dispositivos tecnológicos? }\end{array}$ & Estudios & Sociales & $\begin{array}{c}\text { Entretenimiento/ } \\
\text { Juegos }\end{array}$ & Música/Videos \\
\hline frecuencia & 65 & 65 & 27 & 44 \\
$\%$ & 70,65 & 70,65 & 29,35 & 47,83 \\
\hline
\end{tabular}

Figura 4. ¿Principalmente para que usa los dispositivos tecnológicos?

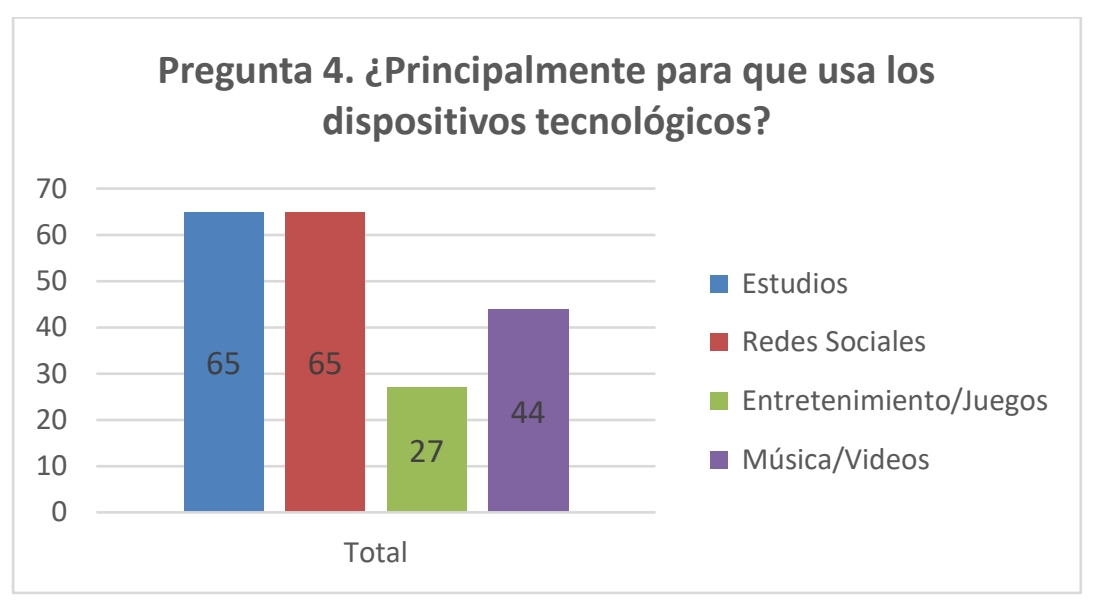

Análisis pregunta 4. ¿Principalmente para que usa los dispositivos tecnológicos?

La tabulación de datos de la pregunta cuatro arrojos que los estudiantes principalmente hacen uso de los dispositivos tecnológicos son los siguientes tenemos con un 70,65\% que utilizan para las redes sociales de igual manera para sus estudios, con un 47,83\% para música/video y finalmente con un 29,35\% para entretenimiento/juegos, es decir que utilizan más del $50 \%$ de estudiantes para las redes sociales y estudios, y con un porcentaje mínimo que es del $29,35 \%$ es para escuchar música. 
Tabla 5: ¿Frecuenta el uso de dispositivos tecnológicos con acceso a internet?

\begin{tabular}{ccc}
\hline $\begin{array}{c}\text { ¿Frecuenta el uso de dispositivos } \\
\text { tecnológicos con acceso a internet? }\end{array}$ & frecuencia & $\boldsymbol{\%}$ \\
\hline $\mathrm{No}$ & 11 & $11,96 \%$ \\
$\mathrm{Si}$ & 81 & $88,04 \%$ \\
Total general & $\mathbf{9 2}$ & $\mathbf{1 0 0 , 0 0 \%}$ \\
\hline
\end{tabular}

Figura 5. ¿Frecuenta el uso de dispositivos tecnológicos con acceso a internet?

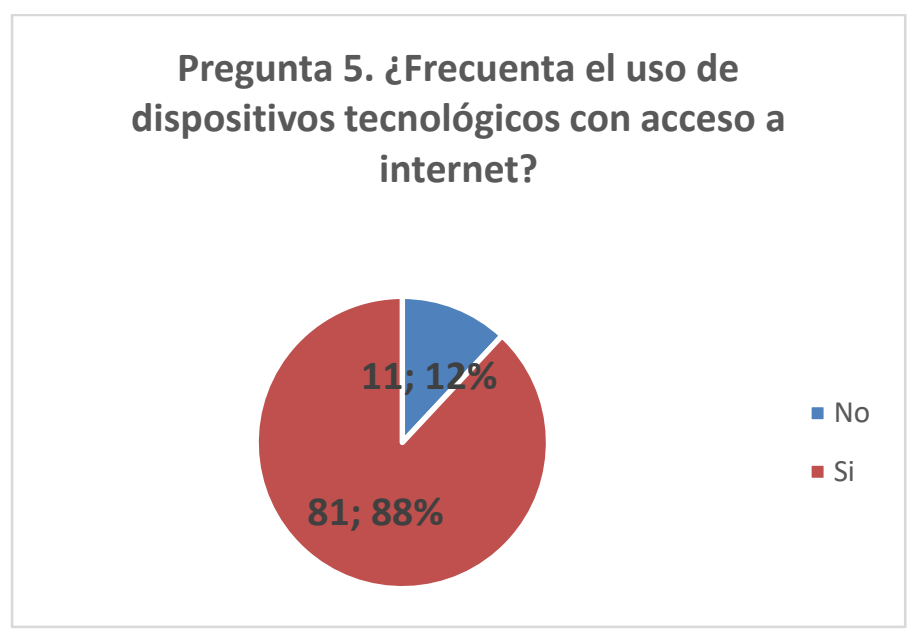

Análisis pregunta 5. ¿Frecuenta el uso de dispositivos tecnológicos con acceso a internet? En la pregunta cinco se puede mencionar que el $88 \%$ de los estudiantes cuenta con al menos un dispositivo tecnológico con acceso a internet de los cuales utilizan con mayor frecuencia para las redes sociales y estudios, con menor frecuencia para entretenimiento y con un $12 \%$ de los estudiantes no cuentan con un dispositivo tecnológico que tenga acceso a internet, es decir, que los estudiantes que utilizan de 1-2 horas el dispositivo tecnológico son los que no tienen un dispositivo que tenga acceso a internet.

Tabla 6. ¿En qué lugar usa con más frecuencia dispositivos tecnológicos?

\begin{tabular}{ccccc}
\hline $\begin{array}{c}\text { ·EEn qué lugar usa con más } \\
\text { frecuencia dispositivos } \\
\text { tecnológicos? }\end{array}$ & Casa & Universidad & Ciber Café & $\begin{array}{c}\text { Otros (en } \\
\text { cualquier } \\
\text { lugar) }\end{array}$ \\
\hline $\mathrm{f}$ & 81 & 41 & 2 & 3 \\
$\%$ & 88,04 & 44,57 & 2,17 & 3,26 \\
\hline
\end{tabular}


Figura 6. ¿En qué lugar usa con más frecuencia dispositivos tecnológicos?

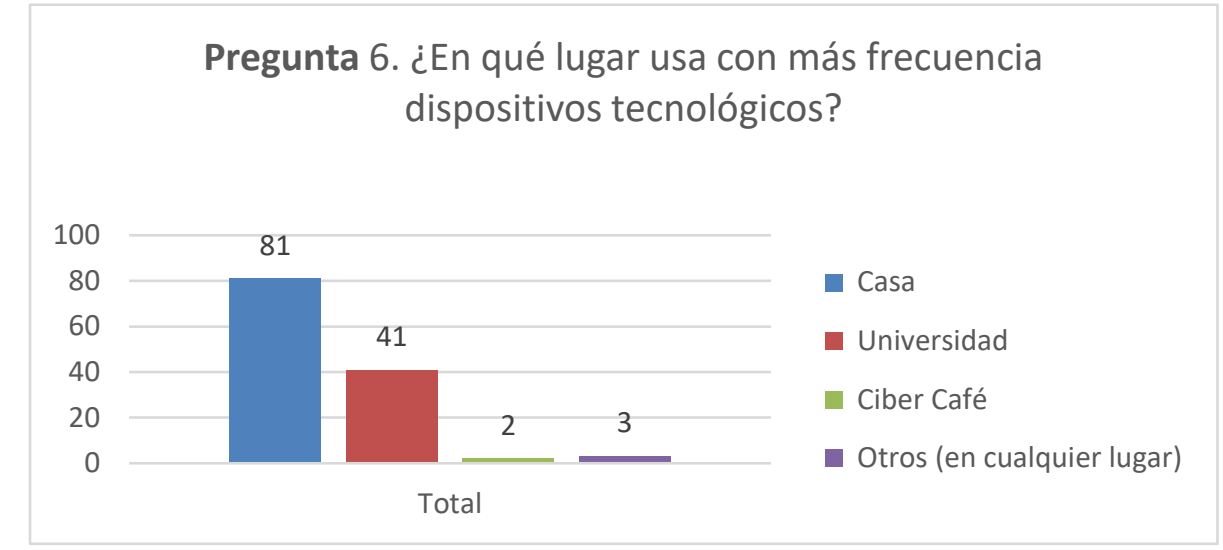

Análisis pregunta 6. ¿En qué lugar usa con más frecuencia dispositivos tecnológicos?

En esta pregunta se muestra que los estudiantes usan más los dispositivos electrónicos en casa con un $88,02 \%$ de un total de 92 encuestas, mientras que el segundo lugar lo ocupa la universidad con un 44,57\%, el ciber café lo frecuentan muy pocos y en la opción otros corresponden a cualquier lugar posible.

Tabla 7. ¿Cree usted que podría soportar una semana sin usar un dispositivo tecnológico

¿Cree usted que podría soportar una semana sin usar un dispositivo tecnológico?

$\begin{array}{lcc}\text { No } & 39 & 42,39 \% \\ \mathrm{Si} & 53 & 57,61 \% \\ \text { al general } & 92 & 100,00 \%\end{array}$

Figura 7. ¿Cree usted que podría soportar una semana sin usar un dispositivo

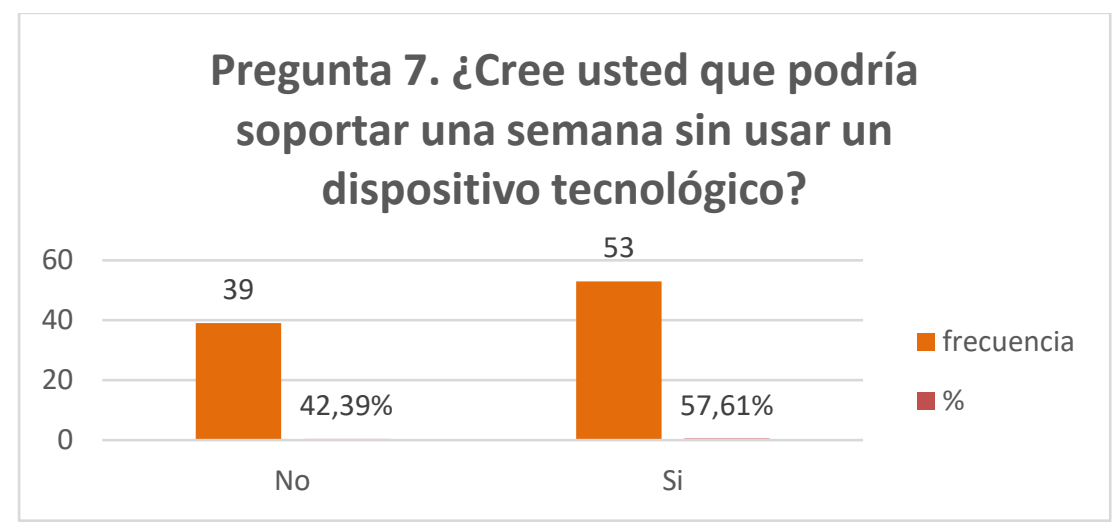


Análisis pregunta 7. ¿Cree usted que podría soportar una semana sin usar un dispositivo tecnológico?

Mediante la información recopilada por medio de la encuesta dirigida a los estudiantes y luego la tabulación de los datos se puede verificar que un $42,39 \%$ de estudiantes dicen que no podrían soportar una semana sin usar un dispositivo tecnológico y un $57,61 \%$ dicen que sí podrían soportar sin una semana sin usar el dispositivo tecnológico.

Tabla 8. ¿Ha tenido problemas por el uso constante de dispositivos tecnológicos?

\begin{tabular}{ccc}
\hline $\begin{array}{c}\text { ¿Ha tenido problemas por el uso constante de } \\
\text { dispositivos tecnológicos? }\end{array}$ & frecuencia & $\%$ \\
\hline No & 65 & $70,65 \%$ \\
Si & 27 & $29,35 \%$ \\
Total general & 92 & $100,00 \%$ \\
\hline
\end{tabular}

Figura 8. ¿Ha tenido problemas por el uso constante de dispositivos tecnológicos?

\section{Pregunta 8. ¿Ha tenido problemas por el uso constante de dispositivos tecnológicos?}

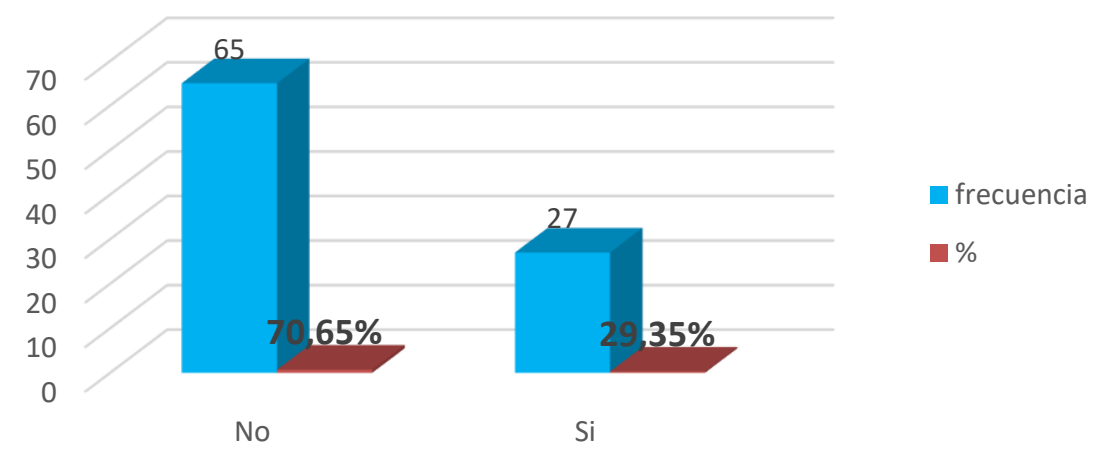

Análisis pregunta 8 ¿Ha tenido problemas por el uso constante de dispositivos tecnológicos? Debido a la encuesta realizada a los estudiantes obtuvimos un 70,65\% de la población dice que no han tenido ningún problema por el uso constante del dispositivo tecnológico y el 29,35\% dice que, si han tenido problemas con el uso constante del dispositivo tecnológico, es decir, que mal del $50 \%$ de la población no han tenido ningún inconveniente por el uso de ellos. 
Tabla 9: ¿Qué tipos de problemas ha presentado por el uso de dispositivos tecnológicos?

¿Qué tipos de problemas ha presentado por el uso de dispositivos tecnológicos? Académicos Económicos Familiares Otros

$\begin{array}{lllll}\mathrm{f} & 33 & 12 & 21 & 23\end{array}$

$\begin{array}{llll}\% & 35,87 & 13,04 & 22,83\end{array}$

Figura 9. ¿Qué tipos de problemas ha presentado por el uso de dispositivos tecnológicos?

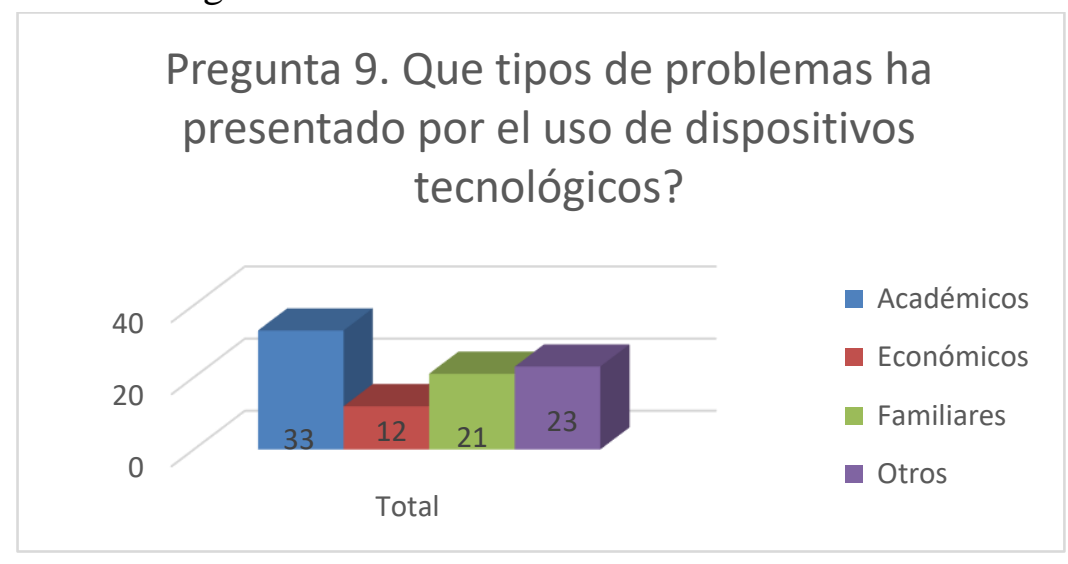

Análisis pregunta 9. ¿Qué tipos de problemas ha presentado por el uso de dispositivos tecnológicos?

Después de observar el grafico podemos afirmar que 33 estudiantes han tenido problemas académicos por el uso de dispositivos tecnológicos esto corresponde a un $35,87 \%$ del total de estudiantes. En otros casos se tiene que el 13,04\% ha tenido problemas económicos y el $23,83 \%$ problemas familiares, en otros problemas tenemos un $25 \%$ que corresponde a problemas de salud, dinero y concentración.

Tabla 10. ¿Cree que el uso de los dispositivos pueda ocasionarle algún tipo de problemas en el futuro?

\begin{tabular}{ccc}
\hline $\begin{array}{c}\text { ¿Cree que el uso de los dispositivos } \\
\text { tecnológicos pueda ocasionarle algún } \\
\text { tipo de problemas en el futuro? }\end{array}$ & frecuencia & $\%$ \\
\hline No & 33 & $35,87 \%$ \\
Si & 59 & $64,13 \%$ \\
Total general & 92 & $100,00 \%$ \\
\hline
\end{tabular}


Figura 10. ¿Cree que el uso de los dispositivos pueda ocasionarle algún tipo de problemas en el futuro?

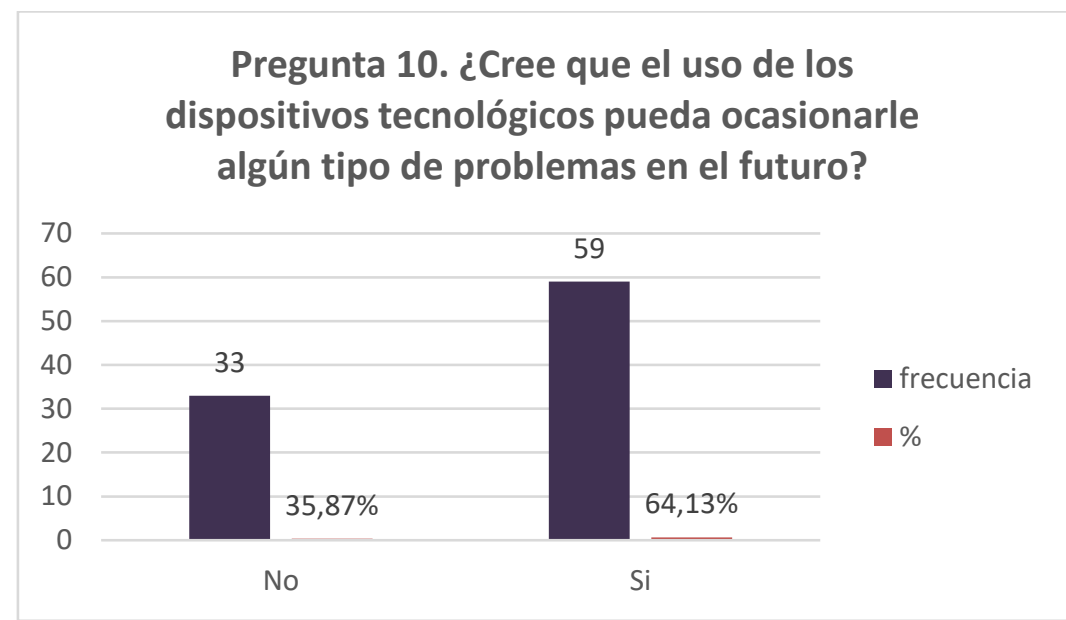

Análisis pregunta 10 ¿Cree que el uso de los dispositivos tecnológicos pueda ocasionarle algún tipo de problemas en el futuro?

Mediante la recolección de datos obtuvimos de los estudiantes, los siguientes resultados de una encuesta lo cual podemos constatar que el 35,87\% de los estudiantes desconocen los posibles problemas que les puedan ocasionar el uso de dispositivos tecnológicos y con un $64,13 \%$ de los estudiantes conocen los riesgos que tiene el uso de dispositivos tecnológicos, es decir, que menos del 50\% no conocen las consecuencias que les traer el uso de dispositivos tecnológicos.

\section{Conclusiones}

- El uso de los dispositivos tecnológicos es esencial para la vida de las personas. Gracias a estos podemos comunicarnos de manera más fácil, agilizan el trabajo y facilita el aprendizaje de varios temas y es así que son indispensables los dispositivos tecnológicos para la vida diaria de las personas.

- La característica de los dispositivos tecnológicos es que ocupa una parte central de la vida de las personas, que utiliza la pantalla del ordenador ya sea para entretenimiento o trabajo, pero descuidando así mismo en cierta manera su salud al estar expuesto a la luz de las pantallas por demasiado tiempo.

- En los datos analizados se pudo constatar que la mayor parte de los adolescentes usan sus dispositivos durante prolongadas horas exponiéndose de esta manera a problemas de visión, académicos por falta de control adecuado. 


\section{Referencias bibliográficas.}

Abece (2014) Como afecta la tecnología en la comunicación familiar. Recuperado el 16 de febrero de 2018, de: http://www.abcdelbebe.com/familia/crianza-en-familia/comoafecta-el-internet-y-la-tecnologia-la-comunicacion-familiar-14022

Becoña, E. (2009). Factores de riesgo y de protección en la adicción a las nuevas tecnologías. En E. Echeburúa, F.J. Labrador y E. Becoña (Eds.), Adicción a las nuevas tecnologías en adolescentes y jóvenes (pp. 77-97). Madrid: Pirámide.

Carbonell, X., Graner, C. (2009). Fomento del uso saludable de las tecnologías de la información y comunicación en familia y escuela. Madrid: Pirámide.

Echeburúa, E. (2012). Factores de riesgo y factores de protección en la adicción a las nuevas tecnologías y redes sociales en jóvenes y adolescentes. Revista Española de Drogodependencias

Echeburúa, E. y Corral, P. de (2010). Adicción a las nuevas tecnologías y a las redes sociales en jóvenes: un nuevo reto. España: R.E.

Espinoza S. (4 de febrero del 2015). Abusos de tecnologías afecta al cerebro. El Comercio, pp.16.

Sura, (2012) Calidad de vida. Recuperado el 16 de febrero de 2018, de: https://www.sura.com/blogs/calidad-de-vida/tecnologia-relaciones-familia.aspx

Salud180 (2017) Enfermedades oculares por el uso de la tecnología. Recuperado el 16 de febrero de 2018, de: http://www.salud180.com/7-enfermedades-oculares-por-uso-dela-tecnologia

Scrib (2016) Uso de la tecnología en la actualidad. Recuperado el 16 de febrero de 2018, de: https://es.scribd.com/doc/21384676/el-uso-de-la-tecnologia-en-la-actualidad.

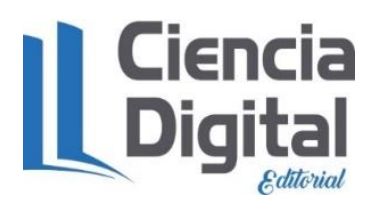




\section{PARA CITAR EL ARTÍCULO INDEXADO.}

Aguilar Reyes, J., Valverde Aguirre, P., Sánchez Vimos, J., \& Altamirano Novillo, T. (2019). Uso de dispositivos tecnológicos de los estudiantes de Fima de la Espoch, carrera de Estadística. Ciencia Digital, 3(3.3), 95-110. https://doi.org/10.33262/cienciadigital.v3i3.3.773

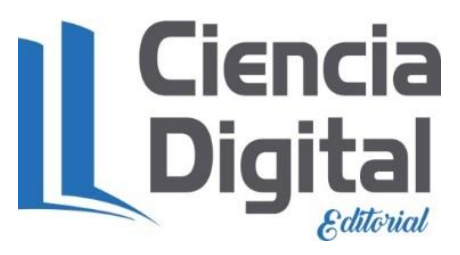

El artículo que se publica es de exclusiva responsabilidad de los autores y no necesariamente reflejan el pensamiento de la Revista Ciencia Digital.

El artículo queda en propiedad de la revista y, por tanto, su publicación parcial y/o total en otro medio tiene que ser autorizado por el director de la Revista Ciencia Digital.
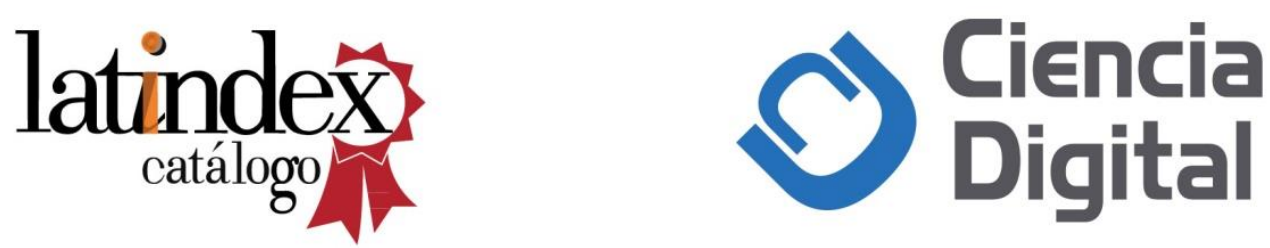